\title{
CAMBIOS HEMODINÁMICOS POR DOPPLER EN FETOS CON RETARDO DEL CRECIMIENTO INTRAUTERINO DE 26-34 SEMANAS A 24 Y 48 HORAS DE LA ADMINISTRACIÓN MATERNA DE BETAMETASONA
}

José L uis Rojas M D*, Carol Gisela Rueda M D**,A Ifredo Rincón M D***, Oscar Gregorio Coronado M D***

\section{Resumen}

Objetivo: identificar cambios en el índice de pulsatilidad (IP) de las arterias umbilical y cerebral media después de aplicar betametasona en pacientes con retardo del crecimiento intrauterino (RCIU) entre 26 y 34 semanas. Métodos: 22 pacientes hospitalizadas con embarazos únicos entre las 26 y 34 semanas asociadas con RCIU, con indicación de maduración pulmonar que no se encontraban en trabajo de parto, recibieron protocolo completo de maduración, toma de doppler fetoplacentario inicial y a las 24 y 48 horas. Resultados: 68,2\% presentaron trastorno hipertensivo del embarazo, 81,8\% (n:18) negaron enfermedad crónica asociada, no se documentaron anomalías fetales mayores ni se sospechó infección fetal. El promedio del IP de la arteria umbilical al ingreso fue 1,62 (DE 0,41) y de la cerebral media 1,97 (DE 0,61). En el doppler de 48 horas se observaron cambios del IP en la umbilical $(p=0.0079)$ y la cerebral media ( $\mathrm{p}=\mathbf{0 . 0 1 4 9}$ ), respecto al basal. Conclusiones: en RCIU entre las semanas 26 y 34 hay variaciones con significación estadística del IP en el doppler de las arterias umbilical y cerebral media que no siempre se asociaron con cambios en la estadificación del doppler actual y no tienen importancia clínica. La hipertensión gestacional asociada puede ser un factor de confusión.

Palabras clave: cambios hemodinámicos, doppler, retardo del crecimiento intrauterino, betametasona. Abreviaturas: IP, índice de pulsatilidad: RCIU, retardo en el crecimiento intrauterino.

\section{HEMODYNAMIC CHANGES DETECTED BY DOPPLER BETWEEN 26-34 WEEKS PREGNANCIES WITH IUGR 24 AND 48 HOURS AFTER BETAMETHASONE ADMINISTRATION}

\section{Abstract}

Objective: to identify changes in the pulsatility index (PI) of the umbilical and middle cerebral arteries after betamethasone administration in pregnancies at 26-34 gestational weeks with intrauterine growth retardation (IUGR). Methods: the study comprised 22 hospitalized women with singleton pregnancies at 26- 34 weeks with IUGR, not in

Fecha recibido: enero 22 de 2015 - Fecha aceptado: febrero 26 de 2015

* Especialista en Medicina Materno Fetal y en Epidemiologia Clínica. Jefe Departamento de Obstetricia, Hospital de San José. Profesor Asociado, Fundación Universitaria de Ciencias de la Salud. Bogotá Colombia.

\footnotetext{
** Residente II Medicina Maternofetal, Fundación Universitaria de Ciencias de la Salud. Bogotá DC, Colombia.

*** Residente IV de Ginecología y Obstetricia, Fundación Universitaria de Ciencias de la Salud. Bogotá DC, Colombia.
} 
labor, with indication of fetal lung maturity enhancement who received a complete treatment protocol and an initial fetoplacental Doppler repeated after 24 and 48 hours. Results: pregnancy-induced hypertension was present in $68.2 \%$, 81.8\% (n: 18) denied having an associated chronic illness, no major fetal anomalies or suspicion of fetal infection were documented. The average PI of the umbilical artery at admission was 1.62 (SD 0.41) and of the middle cerebral artery 1.97 (SD 0.61). PI changes observed in the Doppler performed after 48 hours were $(p=0.0079)$ in the umbilical artery and $(p=0.0149)$ in the middle cerebral artery, compared with baseline PI. Conclusions: there are statistically significant variations of Doppler PI in the umbilical and middle cerebral arteries in IUGR between week 26 and 34 which were not always related with changes of current Doppler stages and have no clinical importance. The associated gestational hypertension may constitute a confounding factor.

Key words: hemodynamic changes, Doppler, intrauterine growth retardation, betamethasone.

\section{Introducción}

Los fetos con RCIU se definen por una de las siguientes características: peso estimado fetal menor al percentil 10 asociado con doppler fetoplacentario alterado, peso estimado fetal menor al percentil 3, 0 circunferencia abdominal con dos desviaciones estándar por debajo del esperado para la edad gestacional; la incidencia de R CIU es variable teniendo en cuenta las distintas poblaciones, se estima que entre 4 y $8 \%$ de todos los recién nacidos en los países desarrollados presentan RCIU y entre $6 \%$ y $30 \%$ en aquellos en desarrollo. ${ }^{1-3}$

El uso de glucocorticoides en el contexto de la paciente con riesgo de parto pretérmino es de manejo frecuente y ha demostrado beneficios para el neonato en su periodo posnatal; sin embargo, en el contexto de la paciente con RCIU son muchos los interrogantes que quedan por resolver. ${ }^{4-6}$

Existen diferentes autores como R otmensch, Schaap, Simchen, M iller y R obertson que han investigado la relación existente entre el uso de corticoides y los efectos hemodinámicos evaluados por doppler feto placentario y el comportamiento fetal evaluado por perfil biofísico fetal (PBF). ${ }^{6}$ A lgunos han observado deterioro transitorio en el PBF a expensa de movimientos respiratorios y corporales asociados o no con cambios hemodinámicos los cuales incluyen vasodilatación transitoria de arteria umbilical y en otros casos incremento en la resistencia vascular de la misma. Sin embargo otros autores no han visto ningún cambio y algunos observaron asociación entre los cambios hemodinámicos y los resultados perinatal es como estancia en $\mathrm{UCI}$, discapacidad y mortalidad.

Las diferencias observadas por los distintos autores podrían estar relacionadas con el momento de la administración del medicamento, cuando se hace la medición (doppler) y la asociación de estos posibles cambios con la etiología del RCIU. . $^{4-6}$

L as pacientes con R CIU están expuestas a riesgos adicionales al que tienen aquellas sin esta condición y es el parto pretérmino en muchos casos el momento de nacimiento para estos fetos. ${ }^{7,8}$ Para estratificar estas pacientes se utiliza el doppler fetoplacentario y podría ser un marcador de bienestar 0 deterioro fetal. ${ }^{8}$ Conociendo la acción vasodilatadora de la betametasona, son contradictorios los efectos final es sobre el doppler fetoplacentario y los resultados perinatal es. ${ }^{8}$ Teniendo en cuenta el amplio uso de los corticoides en el contexto de la maduración pulmonar en nuestro medio, se describirán los cambios hemodinámicos después de la aplicación de betametasona reflejados en el doppler fetoplacentario de pacientes con RCIU.

\section{Métodos}

El grupo consistía en mujeres embarazadas entre 26 y 34 semanas con gestación única, diagnóstico de 
RCIU e indicación para nacimiento inminente, que recibieron betametasona 12 mg intramuscular cada 24 horas, dos dosis, como parte del protocolo de maduración pulmonar. Ingresaron a los Hospitales de San José e Infantil U niversitario de San José de Bogotá DC, Colombia, aquellas con doppler previo al inicio de la betametasona. Los estudios ecográficos fueron real izados en las unidades maternofetal es del sitio de ingreso por el instructor de medicina materno fetal de turno y utilizando los equipos M edison Accuvix v10 y Toshiba Xario.

La evaluación del doppler se hizo al inicio del estudio a las 24 y 48 horas posteriores a la aplicación de betametasona. Se estimó el IP de las arterias umbilical cerebral media. Se usó la clasificación de Vall Hebrón real izando doppler venoso en los casos indicados (alteración del IP de las arterias umbilical o cerebral media). Para la primera se localizó el sitio que cumpliera con ser un asa libre del cordón con angulación del doppler a 0 grados y para la cerebral media el tercio medio de la misma, a través de ecografía transabdominal concomitante con doppler color para una mejor identificación del sitio de medición. ${ }^{9}$

EI IP de las arterias se calculó en forma automática por el equipo utilizado. El peso estimado fetal y su percentil fueron comparados con las tablas Hadlock, de esta forma se consideró RCIU cuando el percentil fue menor de 30 entre 3 y 10 con alteración del doppler según la clasificación de Vall H ebrón o cuando la circunferencia abdominal estaba dos desviaciones estándar por debajo de lo esperado para la edad gestacional. ${ }^{10,11}$

$L$ as pacientes fueron seguidas hasta el al ta hospitalaria. $L$ as variables perinatales reportadas incluyeron A P. GAR al minuto, peso al nacer, ingreso a U CI neonatal y requerimiento de soporte ventilatorio e inotrópico del recién nacido. Se contó con el consentimiento informado de cada una de las pacientes como parte de la exigencia del comité de ética médica de la FU CS. En el análisis estadístico las variables cualitativas se resumen con frecuencias absolutas y relativas, las cuantitativas con medidas de tendencia central y dispersión. Se uti- lizó la prueba de Friedman para evaluar los cambios en el IP. Se usó el software Stata 13.

\section{Resultados}

Se analizaron 22 casos, la edad promedio de las pacientes fue 27,4 años (rango 17-33), la edad gestacional promedio fue 29,6 semanas (rango 26,3 - 33). La edad gestacional promedio del parto fue 32,2 semanas (rangos 27-39). El 68,2\% (n:15) presentaba trastorno hipertensivo asociado con el embarazo. Negaron enfermedad crónica el $81,8 \%$ (n: 18) y ninguna refirió exposición a tabaco. No se documentaron anomalías fetales mayores ni se sospechó infección fetal (Tabla 1).

EI promedio del IP de la arteria umbilical al ingreso fue $1,62(\mathrm{DE} 0,41)$ y el de la arteria cerebral media 1,97 (DE 0,61). D entro de los resul tados la mayoría fueron doppler II (36.4\%), una paciente con flujo ausente y otra con flujo reverso (Tabla 2 ).

En el seguimiento del doppler a las 24 y 48 horas, hubo cambios tanto en el IP umbilical ( $p=0.0079)$ como de la cerebral media ( $p=0.0149)$, respecto al basal, que se observaron a las 48 horas. Predominaron las categorías I y II del doppler a las 24 y 48 horas, no hubo doppler $\checkmark$ que pudiera hablar de deterioro, tampoco se observó flujo reverso pero se identificaron dos casos a las 24 horas y cuatro casos a las 48 horas de flujo ausente (Tabla 2). Se obtuvieron resultados perinatales en 17 pacientes, con pérdida de datos en cinco casos que requirieron remisión para el nacimiento.

La vía del parto fue la alta en trece (76.5\%). EI peso promedio al nacimiento 1.417 gramos (DE 791gr), de estos el $64,7 \%$ de sexo masculino (13). Se reportaron $5(29,4 \%)$ muertes neonatales intrahospitalarias, de estas solo uno nació muerto, tratándose de una paciente con una gestación de 27 semanas en el momento del diagnóstico y 29 semanas al nacimiento, con doppler inicial categoría 3, control posterior 4, con peso al nacer de 450 gramos. Requirieron atención especial incluido soporte ventilatorio $8(47 \%)$ e inotrópico 4 $(23,5 \%)$ (Tabla 3$)$. 


\section{Tabla I. Caracteristicas basales (n:22)}

\begin{tabular}{c} 
Edad materna (DE) \\
\hline mínima-máxima
\end{tabular}
$27,4(6,4)$

\section{Estrato socioeconómico*, n (\%)}

\begin{tabular}{|c|c|}
\hline 1 & I $(4,6)$ \\
\hline 2 & $9(40,9)$ \\
\hline 3 & $12(54,6)$ \\
\hline \multicolumn{2}{|l|}{ Procedencia } \\
\hline Bogotá (\%) & $21(95,5)$ \\
\hline Girardot (\%) & I $(4,6)$ \\
\hline Cigarrillo, n (\%) & $0(0)$ \\
\hline $\begin{array}{l}\text { Edad gestacional al diagnóstico, semanas- } \\
\text { días, promedio (DE) }\end{array}$ & $29,6(2,2)$ \\
\hline $\min$ & 26,3 \\
\hline $\max$ & 33 \\
\hline $\begin{array}{l}\text { †Edad gestacional al momento del parto } \\
\text { (DE) n: } 20\end{array}$ & $32,2(3,3)$ \\
\hline $\min$ & 27 \\
\hline $\max$ & 39 \\
\hline \multicolumn{2}{|l|}{$\begin{array}{l}\text { Trastornos hipertensivos asociados con el } \\
\text { embarazo (\%) }\end{array}$} \\
\hline ninguna & $7(3 \mid, 8)$ \\
\hline hipertensión gestacional & $2(9,1)$ \\
\hline preeclampsia no severa & $3(13,6)$ \\
\hline preeclampsia severa & $9(40,9)$ \\
\hline HTA crónica & I $(4,6)$ \\
\hline \multicolumn{2}{|l|}{ Enfermedad materna preexistente (\%) } \\
\hline ninguna & $18(81,8)$ \\
\hline diabetes mellitus & I $(4,6)$ \\
\hline hiper o hipotiroidismo & I $(4,6)$ \\
\hline trastornos inmunológicos & I $(4,6)$ \\
\hline infección & I $(4,6)$ \\
\hline \multicolumn{2}{|l|}{ Hallazgos fetales anormales (\%) } \\
\hline ninguno & $21(95,5)$ \\
\hline Alteraciones gastrointestinales & I $(4,6)$ \\
\hline \multicolumn{2}{|l|}{ Infección fetal (\%) } \\
\hline ninguna & $22(100)$ \\
\hline
\end{tabular}

* Clasificación del inmueble donde vive el paciente asociada con el pago de los servicios públicos. † Aclarada información de nacimiento para dos pacientes.

\section{Tabla 2. Cambios hemodinámicos}

Ingreso, 24 horas, 48 horas, $n: 22$ n: 22 n: 22 \begin{tabular}{l|l|l|l}
$\begin{array}{l}\text { IP Arteria umbilical , } \\
\text { promedio (DE) }\end{array}$ & $\mathrm{I}, 62(0,4 \mathrm{I})$ & $\mathrm{I}, 63(0,69)$ & $\mathrm{I}, 44(0,74)$ \\
\hline
\end{tabular}

\begin{tabular}{l|l|l}
\hline $1,97(0,61)$ & $2,00(0,53)$ & $1,86(0,37)$
\end{tabular}

media, promedio (DE)

\begin{tabular}{|l|l|l|}
\hline & \\
\hline
\end{tabular}

Doppler inicial, (\%)

\begin{tabular}{|c|c|c|c|}
\hline I & $2,(9, I)$ & $6,(27,3)$ & $3,(13,6)$ \\
\hline II & $8,(36,4)$ & $10,(45,5)$ & $13,(59, I)$ \\
\hline III & $6,(27,3)$ & $3,(13,6)$ & $2,(9, I)$ \\
\hline IV & $6,(27,3)$ & $2,(9, I)$ & $2,(9, I)$ \\
\hline V & - & $\mathrm{I},(4,6)$ & $2,(9, \mathrm{I})$ \\
\hline Flujo ausente, (\%) & $\mathrm{I},(4,6)$ & $2,(9, \mathrm{I})$ & $4,(18,2)$ \\
\hline Flujo reverso, (\%) & $\mathrm{I},(4,6)$ & - & - \\
\hline
\end{tabular}

\section{Tabla 3. Resultados perinatales, $\mathrm{n}: 17$}

\begin{tabular}{|c|c|}
\hline Vía del parto n:I7 & \\
\hline vaginal, (\%) & $4(23,5)$ \\
\hline cesárea, (\%) & $13(76,5)$ \\
\hline Sexo masculino & II $(64,7)$ \\
\hline $\begin{array}{l}\text { Peso del recién nacido, } \\
\text { gramos, promedio (DE) }\end{array}$ & 1.417 (79I) \\
\hline \multicolumn{2}{|l|}{ Apgar al minuto, (\%) } \\
\hline 0 & I $(5,9)$ \\
\hline I & I $(5,9)$ \\
\hline 2 & I $(5,9)$ \\
\hline 3 & 0 \\
\hline 4 & I $(5,9)$ \\
\hline 5 & 0 \\
\hline 6 & I $(5,9)$ \\
\hline 7 & $3(17,6)$ \\
\hline 8 & $5(29,4)$ \\
\hline 9 & 0 \\
\hline 10 & 0 \\
\hline Soporte ventilatorio, (\%) & $8(47)$ \\
\hline Soporte inotrópico, (\%) & $4(23,5)$ \\
\hline $\begin{array}{l}\text { Muerte neonatal } \\
\text { intrahospitalaria, (\%) }\end{array}$ & $5(29,4)$ \\
\hline \multicolumn{2}{|l|}{ pH del cordón, (\%) } \\
\hline$<7,18$ & 0 \\
\hline 7,18 a 7,25 & $3(17,6)$ \\
\hline$>7,25$ & I $(5,9)$ \\
\hline No se determinó & $13(76,5)$ \\
\hline
\end{tabular}




\section{Disc usión}

El presente estudio evaluó los cambios en el IP de las arterias umbilical y cerebral media a las 24 y 48 horas del inicio de la betametasona. Como otros autores describen protocolos de seguimiento con toma del doppler inicial en los primeros tres días y el segundo entre el 5 y 7 , no es posible comparar nuestros hallazgos con lo reportado en la literatura.

No encontramos cambios significativos del IP de las arterias mencionadas en el doppler de las 24 horas; pero sí se documentaron en el doppler de las 48 horas. Estos cambios aunque tuvieron significancia estadística, los consideramos sin importancia en la clínica debido a que no modifican la conducta. A demás no es posible asociarlos con el uso de la betametasona por las distintas variables que podrían interactuar en el cambio observado. A utores como N ozaki y col. ${ }^{12}$ reportan en pacientes con flujo diastólico ausente cambios a las 24 horas de aplicar betametasona, pero no es posible compararnos debido a que incluimos los doppler de I a IV y solo una paciente no tenía flujo al inicio, que después se pudo documentar.

La revisión de la literatura publicada por M ulder y col. revela cambios en la arteria umbilical en 6 de 17 artículos, describiéndolo como una mejoría del doppler de la arteria umbilical después de la aplicación de betametasona sin poder atribuirlo a la misma. 0 tros autores como A. Thuring y col. ${ }^{13}$ encontraron cambios en el IP de la arteria umbilical y el ductus venoso, no así en la cerebral media, que no modificaron la circulación feto placentaria dentro de lo que concluyeron. Vale la pena señal ar que los cambios en los IP fueron observados a las 48 horas y se desconoce su duración.

Con los resultados perinatales no es posible eval uar de manera directa 0 indirecta la asociación con el IP y sugiere diferenciar la respuesta a la betametasona en las distintas categorías de doppler. Los porcentajes de soporte ventilatorio (47\%) 0 inotrópico $(23.5 \%)$ en nuestro estudio son inferiores al de otros autores como Robertson y col. (65\%). Es probable que sea por la inclusión de las distintas categorías de doppler. ${ }^{5}$
Dentro de las fortalezas del estudio están las tomas de doppler realizadas por personal entrenado, con experiencia y fueron correlacionadas por distintos observadores. Limitó el estudio la poca disponibilidad en la unidad neonatal quellevó a una pérdida importante de pacientes y la cantidad de variables que podrían afectar a los resultados del doppler.

\section{Conclusión}

Las paciente con $\mathrm{RCIU}$ entre las 26 y 34 semanas que recibieron betametasona como parte de protocolo de maduración, presentaron cambios significativos en el IP de las arterias umbilical y cerebral media a las 48 horas de observación sin que represente importancia clínica.

\section{Referencias}

1. Resnik R. Intrauterine growth restriction. Obstet Gynecol. 2002; 99(3):490-6

2. Tan TY, Yeo GS. Intrauterine growth restriction. Curr Opin Obstet Gynecol. 2005; 17(2):135-42.

3. M andruzzato $G, A$ ntsaklis A, B otet $F$, Chervenak FA, Figueras $F$, G runebaum A, et al. Intrauterine restriction (IUGR). J Perinat M ed. 2008; 36(4):277-81.

4. Mulder EJ, de Heus R, Visser GH. A ntenatal corticosteroid therapy: short-term effects on fetal behaviour and haemodynamics. Semin Fetal N eonatal M ed. 2009; 14(3):151-6

5. Robertson MC, M urila F, Tong S, Baker LS, Yu VY, Wallace EM. Predicting perinatal outcome through changes in umbilical artery Doppler studies after antenatal corticosteroids in the growth-restricted fetus. Obstet Gynecol. $2009 \mathrm{M}$ ar; 113(3):636-40.

6. Miller SL, Chai M, Loose J, Castillo-M elendez M, Walker DW, Jenkin G, et al. The effects of maternal betamethasone administration on the intrauterine growthrestricted fetus. Endocrinology. 2007; 148(3):1288-95.

7. Chauhan SP, Gupta LM, Hendrix NW, Berghella V. Intrauterine growth restriction: comparison of A merican College of Obstetricians and Gynecologists practice bulletin with other national guidelines. Am J Obstet Gynecol. 2009 A pr;200(4):409.el-6.

8. Ferrazzi E, Bozzo M, Rigano S, B ellotti M, M orabito A, Pardi G, et al. Temporal sequence of abnormal Doppler changes in the peripheral and central circulatory systems of the severely growth-restricted fetus. UItrasound Obstet Gynecol. 2002; 19(2):140-6.

9. Maulik D. Fetal growth compromise: definitions, standards, and classification. Clin Obstet Gynecol. 2006; 49(2):214-8

10. Maulik D. Fetal growth restriction: the etiology. Clin Obstet Gynecol. 2006; 49(2):228-35.

11. Maulik D, Frances Evans J, Ragolia L. Fetal growth restriction: pathogenic mechanisms. Clin Obstet Gynecol. 2006; 49(2):219-27.

12. N ozaki A M , Francisco RP, Fonseca ES, M iyadahira S, Zugaib M . Fetal hemodynamic changes following maternal betamethasone administration in pregnancies with fetal growth restriction and absent end-diastolic flow in the umbilical artery. Acta Obstet Gynecol Scand. 2009; 88(3):350-4.

13. Thuring $A, M$ alcus $P, M$ arsal $K$. Effect of maternal betamethasone on fetal and uteroplacental blood flow velocity waveforms. Ultrasound O bstet Gynecol. 2011; 37(6):668-72. 